\title{
SIMULACIÓN UNIDIMENSIONAL DE ESFUERZOS RESIDUALES DE SECADO EN MADERA DE PINUS RADIATA
}

\author{
C. H. Salinas ${ }^{1}$, C. A. Chavéz ${ }^{1}$, R. A. Ananias ${ }^{2}$ \\ ${ }^{1}$ Departamento de Ingeniería Mecánica, Universidad del Bío-Bío (casali@ ubiobio.cl) \\ ${ }^{2}$ Departamento de Ingeniería en Maderas, Universidad del Bío-Bío
}

Resumen. El trabajo tiene por objetivo estudiar en forma experimental y numérica el problema de los esfuerzos durante el proceso de secado de madera sólida. Para efectos de validación se realizan experimentos de secado convencional isotérmico de piezas de madera de Pinus radiata de 47x101x81 (mm) caracterizado por una temperatura de bulbo seco y húmedo igual a 44 y $36\left({ }^{\circ} \mathrm{C}\right)$, respectivamente. En particular, se modelan efectos de deformación por contracción libre y creep por mecano sorción recurrentes de los gradientes de humedad simulados en base al concepto de difusión efectiva. El modelo matemático resultante es del tipo diferencial parcial no lineal de segundo orden con coeficientes variables, integrado numéricamente a través del Método de Volúmenes de Control Basado en Elementos Finitos (CVFEM). La simulación nuestra una efectiva modelación de los fenómenos estudiados a través de curvas de secado, distribuciones de humedad y esfuerzos mecánicos.

Palabras claves: Madera, secado, esfuerzo, humedad, difusión.

\section{INTRODUCCION}

La deformación asociada a los esfuerzos durante un proceso de secado puede ser caracterizada por cinco contribuciones (Pang 2000): 1) Contracción libre, provocada por disminución del contenido de humedad $(\mathrm{CH})$ bajo el punto de saturación de la fibra; 2) Deformación por relación instantánea de esfuerzo/deformación; 3) Sorción mecánica; 4) Deformación por fuerzas sostenidas por un lapso de tiempo; 5) Deformación por dilatación térmica. Lo anterior, sugiere un comportamiento visco-elástico de la madera sensible a los cambios de $\mathrm{CH}$, la temperatura, la sorción mecánica y cargas permanente

Autores como McMillen (1963), Ormarsson et al. (1998), Ferguson (1998) y Pang (2000), han dado las directrices metodológicas para la modelación unidimensional de esfuerzos y deformaciones durante el proceso de secado. En general, los autores coinciden en una distribución sinusoidal de los esfuerzos transitorios del secado, con distribución semi opuesta desde la superficie hacia el interior de la madera. Modelos bidimensionales y tridimensionales, son dados por Stevensson and Martensson (2002), Kang et al. (2004), Rémond y Perre (2008) y Ormarsson et al. (2009). 
La modelación del transporte de humedad al interior de la madera es realizada a través de un enfoque difusivo clásico del tipo dado por Burr y Stamm (1947), recientemente discutido por Chen (2007). Métodos basados en este enfoque han sido usados tradicionalmente para simulaciones de secado de madera de coníferas por debajo del punto de saturación de la fibra $(P S F)$, debido a que en este rango es dominante el transporte difusivo. Sobre el PSF, estos modelos difusivos presentan restricciones debido a la dominancia de otros fenómenos, tales como capilaridad y permeabilidad (Keey et al. 2000). No obstante, los modelos difusivos pueden ser utilizados más allá del rango higroscópico en base a un coeficiente efectivo de difusión del agua $(D)$, como implementado por Hukka (1999) y Rozas et al. (2009) para coníferas, y aplicado en particular, al Pinus radiata por Gatica et al. (2011) y Salinas et al. (2012).

Este trabajo tiene como objetivos determinar los esfuerzos residuales de secado de madera de Pinus radiata, obtenidos experimentalmente y correlacionarlos con simulaciones numéricas a través de CVFEM (Baliga y Patankar, 1980), previa determinación de coeficientes de difusión efectiva $D$ y convección de masa $h_{m}$, en base una metodología inversa.

\section{EXPERIMENTOS}

Se realizaron de secado isotérmico de Pinus radiata en una cámara de clima (Heraeus Votsch model Virk 150), en la cual recircula aire húmedo a una velocidad promedio igual a 1.6 $(\mathrm{m} / \mathrm{s})$ acondicionado a una temperatura bulbo seco y húmedo igual a 44 y $36{ }^{\circ} \mathrm{C}$, respectivamente. Los parámetros de operación fueron monitoreados a través de un sistema de adquisición de datos (figura 1). Los ensayos consistieron en el secado de seis probetas de 47x101x81 $(\mathrm{mm})$, denominadas como $\mathrm{P}_{\mathrm{i}}$, con $\mathrm{i}=1, \ldots 6$, corte tangencial, respectivamente. Las probetas fueron seleccionadas de árboles de Pinus radiata de 20 años de edad crecidas en la región del Bío-Bío - Chile de acuerdo a la normas chilena INN 1984 y 1986. Para favorecer el flujo unidireccional humedad $\left(\mathrm{q}_{\mathrm{M}}\right)$ se sellaron las probetas con silicona y papel de aluminio dejando libres las dos caras contrapuestas. Esto para propiciar que el flujo $\mathrm{q}_{\mathrm{M}}$ sea normal al flujo del ambiente de secado, ver figuras $2 \mathrm{a}$.

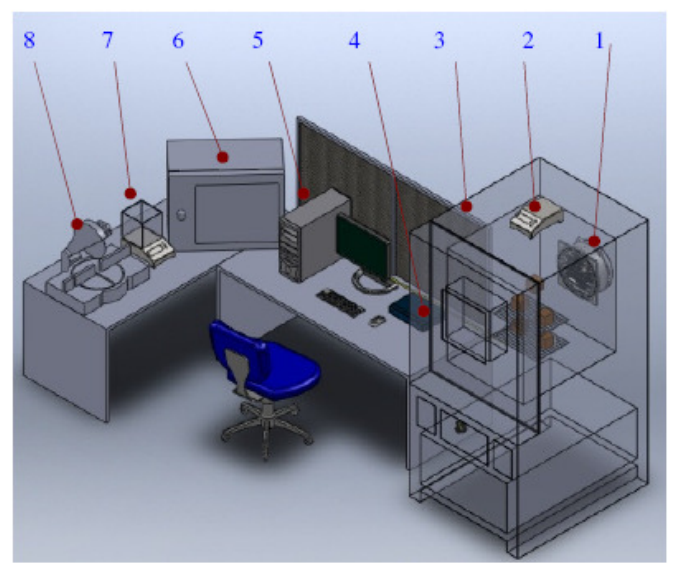

Figura 1. Diagram of experimental equipment: 1) Fan, 2) digital balance (A\&D model GF-4000), 3) climate chamber (Heraeus Votsch model Virk 150), 4) data acquisition system (Fluke model Hydra II), 5) computer, 6) dried-oven (Memmert model U-15), 7) precision balance (Boeckel model BPB32), and 8) sawmill (Makita model LS1040). 
El ensayo contempló dos etapas. La primera etapa consistió en el secado de la probeta $\mathrm{P}_{1}$ a fin de obtener una curva de secado y seleccionar los tiempos de secado con los cuales se realizarían las evaluaciones de distribución de humedades y tensiones residuales.

Las curvas de secado se obtuvieron por el método gravimétrico, según norma chilena INN (1984), siendo los $\mathrm{CH}$ determinados con auxilio de una balanza de precisión (Boeckel model BPB32) y un horno de secado (Memmert model U-15).

$$
C H=\left(\frac{m_{h}-m_{d}}{m_{d}}\right) \cdot 100 .
$$

Siendo $\mathrm{CH}$ el contenido de humedad (\%), $m_{h}$ la masa de madera húmeda $(\mathrm{kg})$ y $m_{d}$ la masa de madera seca $(\mathrm{kg})$.

En la segunda etapa del ensayo fueron secadas las restantes 5 probetas, $P_{i}$, con $i=2, \ldots 6$. En esta etapa, cada $24(\mathrm{~h})$, una probeta fue retirada del proceso de secado y después de ser liberada de la silicona y del papel aluminio, se midió la magnitud y masa de las secciones obtenidas mediante corte con la máquina seccionadora (Makita modelo LS1040), conforme esquematizado en la figura $2 \mathrm{~b}$ y $2 \mathrm{c}$. Lo anterior, permitió obtener 5 evaluaciones transitorias de distribuciones unidimensionales de $\mathrm{CH}$ (radial y tangencial), determinados a través de la ecuación (1), y tensiones residuales a través de (3-7). Estas últimas para cada método de evaluación (seccionado y dientes-tenedor).
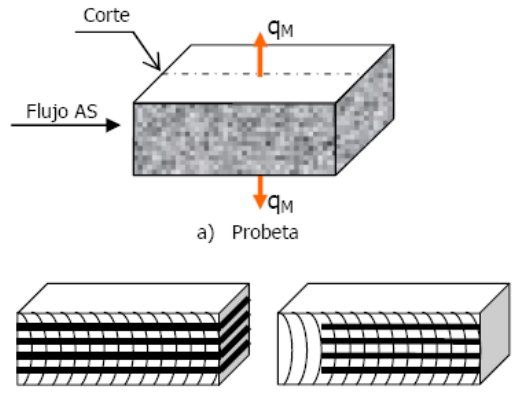

$$
\text { b) Seccionado c) Tenedor }
$$

Figura 2. Esquema de una probeta: a) flujo del AS y $\mathrm{q}_{\mathrm{M}}, \mathrm{b}$ ) seccionado y c) tenedor.

El coeficiente de difusión efectiva $(D)$ fue calculado según el modelo de tipo exponencial propuesto por Hukka (1999), el cual es descrito para un secado isotérmico como

$$
D(C H)=\exp (a+b \cdot \tilde{M}) .
$$

Donde: $\quad \tilde{M}=\min \left(\frac{m_{h}}{m_{d}}, P S F\right)$

$\mathrm{PSF}=0.603-0.001 \cdot T_{d} \quad($ Simpson and Liu, 1997)

$T_{d} \quad$ Temperatura bulbo seco (K).

Los parámetros $a, b$ y el coeficiente de transferencia de masa $h_{m}(\mathrm{~m} / \mathrm{s})$ requerido por el modelo de transporte de $\mathrm{CH}$, fueron determinados a través de un esquema de búsqueda extensiva propuesto por Gatica et al (2011), el cual minimiza la diferencia media entre los valores de $\mathrm{CH}$ simulados y experimentales. Esto es: conocida la distribución transitoria de humedades en la madera, resolver el problema inverso consistente en determinar los parámetros $a$ y $b$ 
de la ecuación (2), además del coeficiente convectivo de transferencia de masa $h_{m}$ requerido en la ecuación (11).

Las deformaciones fueron calculadas según lo propuesto por Moren y SehlstedtPersson (1992), en base a la magnitud de la probeta verde y seca antes de cortas ( $d_{v}$ y $d_{b}$, respectivamente) y la magnitud de cada sección o diente después de cortar $\left(d_{e}\right)$, según ilustrado en figura 3.

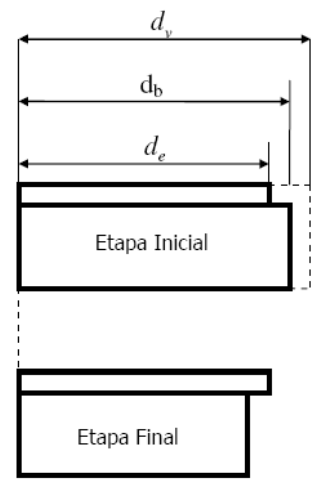

Figura 3. Esquema de una probeta: a) flujo del AS y $\mathrm{q}_{\mathrm{M}}$, b) seccionado y c) tenedor.

Se entiende, en este trabajo, que la deformación total de la madera $\varepsilon_{t}$ es resultado de tres contribuciones parciales: 1) Contracción libre, provocada por disminución del contenido de humedad bajo el punto de saturación de la fibra $\varepsilon_{\alpha}$ (Moisture shrinkage); 2) Deformación por Relación Instantánea de Esfuerzo/Deformación $\varepsilon_{\xi}$ (Elastic Stress Properties); 3) Sorción mecánica $\varepsilon_{m s}$ (Mechano-Sorptive Strain). Esto es:

$$
\varepsilon_{t}=\varepsilon_{\xi}+\varepsilon_{\alpha}+\varepsilon_{m s}
$$

A partir de las magnitudes definidas en la figura 3, cada deformación fue determinada como:

$$
\begin{aligned}
& \varepsilon_{t}=\frac{d_{b}-d_{v}}{d_{v}} . \\
& \varepsilon_{\xi}=\frac{d_{b}-d_{e}}{d_{v}} . \\
& \varepsilon_{\alpha}=\frac{d_{x}-d_{v}}{d_{v}} . \\
& \varepsilon_{m s}=\frac{d_{e}-d_{x}}{d_{v}} .
\end{aligned}
$$

Donde $d_{x}$, es definido como la magnitud de la sección o diente en contracción libre. Es decir, es el valor de magnitud que tendría la sección o diente si fuera secada libremente sin estar formando parte de la probeta, definido en función de $\tilde{M}$ dado por la ecuación (2), PSF (punto de saturación de la fibra) y $\alpha$ (coeficiente de contracción) como: 


$$
d_{x}=d_{v}\left(1-100 * \frac{P S F-\tilde{M}}{P S F} \alpha\right) .
$$

Los esfuerzos residuales fueron determinados de acuerdo a lo propuesto por Pang (2000) a través de la siguiente ecuación:

$$
\sigma=-\xi\left(\frac{d_{b}-d_{e}}{d_{e}}\right)
$$

Donde $\sigma$ esfuerzo $(M P a), \xi$ módulo de elasticidad $(M P a)$ y $d_{e}$ y $d_{b}$ como definidos en la figura 3 .

\section{MODELO MATEMATICO}

De forma análoga a lo implementado por Gatica et al (2011). La ecuación diferencial parcial no lineal de segunda orden, que describe el fenómeno de transporte transitorio unidimensional de contenido de humedad $\mathrm{CH}$, es dada por:

$$
\frac{d C H}{d t}=\frac{\partial}{\partial x}\left(D(C H) \frac{\partial C H}{\partial x}\right)+S \quad 0<x<L .
$$

Donde: $\quad \mathrm{CH}$ Contenido de humedad (\%)

$D$ Coeficiente de difusión efectiva $\left(\mathrm{m}^{2} / \mathrm{s}\right)$.

$S$ Termino fuente $(\mathrm{kg} / \mathrm{s})$.

Sujeta a la condición de contorno de convección:

$$
D \frac{\partial C H}{\partial x}=h_{m}\left(\mathrm{CH}_{\mathrm{s}}-\mathrm{CHE}\right) \text { en } x=0 \text { y } x=L .
$$

El modelo reológico fue propuesto en los trabajos desarrollados por Ferguson (1998), como implementado por Chávez (2009). Este modelo es representado por la ecuación diferencial (12).

$$
\frac{\partial \varepsilon}{\partial t}=\frac{1}{\xi} \frac{\partial \sigma}{\partial t}+(\alpha+m \sigma) \frac{\partial C H}{\partial t}
$$

Donde: $\sigma$ es el esfuerzo (Pa); $\varepsilon$ deformación; $\mathrm{CH}$ es el contenido de humedad (\%), $\alpha$ coeficiente de contracción (1/\%), $m$ es el coeficiente de mecano sorción (1/\%) y $t$ el tiempo $(\mathrm{s})$ :

Al integrar en el tiempo (12) resulta la ecuación para la deformación total:

$$
\varepsilon=\frac{1}{\xi} \sigma+\alpha \Delta C H+m \sigma \Delta C H
$$


Como ha sido descrito anteriormente, el modelo está compuesto por deformaciones del tipo: Elástica $\left(\varepsilon_{\xi}=\frac{1}{\xi} \sigma\right)$, contracción libre $\left(\varepsilon_{\alpha}=\alpha \Delta C H\right)$ y sorción mecánica $\varepsilon_{m s}=m \sigma \Delta C$.

\section{RESULTADOS}

\subsection{Contenidos de Humedad}

La figura 4 muestra resultados simulados de curvas de secado y distribuciones espaciales de humedad comparados con resultados experimentales. Las primeras $30(\mathrm{~h})$ corresponden, principalmente, al proceso de extracción de agua libre con $C H<C H C=60(\%)$. Posteriormente, el frente de secado se desplaza al interior de la madera alcanzando el punto de saturación de la fibra $(P S F=30 \%)$ a las 60 (h) y $72(\mathrm{~h})$, respectivamente. Se puede apreciar que los datos experimentales muestran una tendencia definida, cuya distribución transitoria media es captada por la simulación. Las distribuciones espaciales unidimensionales de $\mathrm{CH}$, son de forma parábola con valores mínimos hacia la superficie de la madera, las cuales presentan leves asimetrías debido a la diferencias psicométricas provocadas por las cubetas de humectación. En términos cuantitativos, se tienen desviaciones medias menores a $1 \%$ en las curvas de secado (figuras $4 \mathrm{a}$ y 4b) y menores de 3\% para distribución espacial de humedad (figuras 4c y 4d).

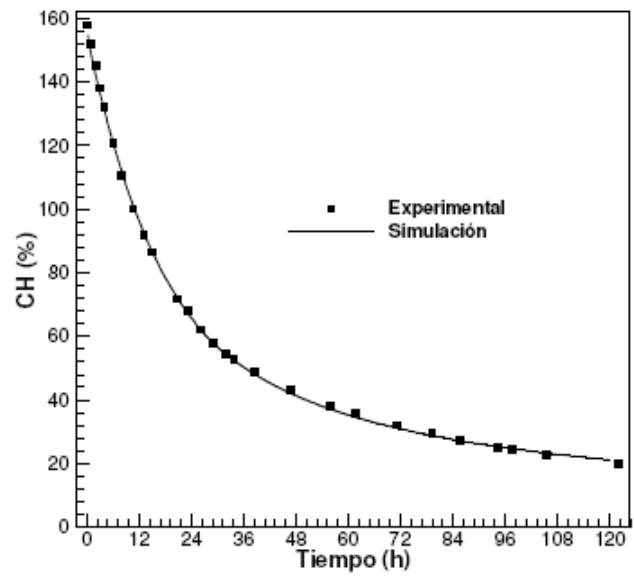

a)

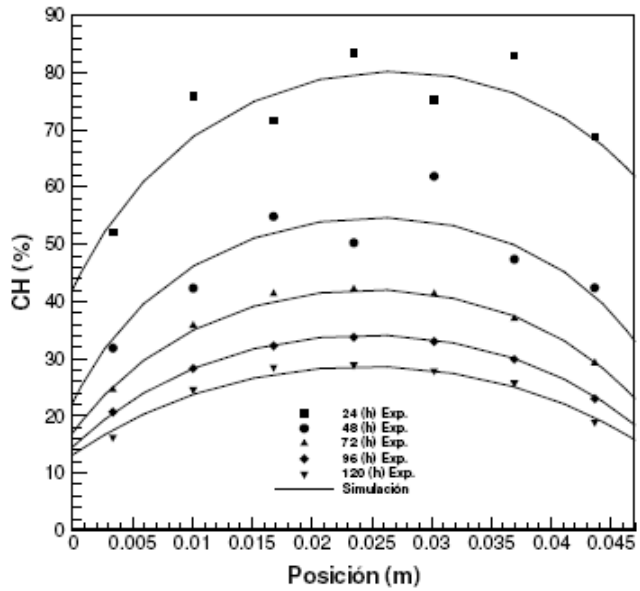

b)

Figura 4. Contenidos de humedad ( $\mathrm{CH})$ : a) Curvas de secado y b) Distribución de $\mathrm{CH}$..

Los resultados de simulación en relación a la búsqueda parámetros $a$ y $b$ que permiten evaluara $D$ y el coeficiente de convección de masa $h_{m}$ son dados en la tabla 1 .

Tabla 1. Parámetros $a, b$ y $\left(h_{m}\right)$.

\begin{tabular}{|c|c|c|}
\hline Rango & \multicolumn{2}{|c|}{ Parámetro } \\
\hline \multirow{3}{*}{ Sobre el $C H C$} & $a$ & -20.60 \\
\cline { 2 - 3 } & $b$ & 10.30 \\
\cline { 2 - 3 } & $h_{m}$ & $2.17^{*} 10^{-7}$ \\
\hline \multirow{3}{*}{ Bajo el $\mathrm{CHC}$} & $a$ & -21.50 \\
\cline { 2 - 3 } & $b$ & 15.30 \\
\cline { 2 - 3 } & $h_{m}$ & $1.92^{*} 10^{-7}$ \\
\hline
\end{tabular}




\subsection{Esfuerzos}

Los resultados experimentales de las de secado de secado son dados en las figuras 5, la cual muestra el comportamiento de los esfuerzos en las probetas evaluadas a las 24, 72 y 120 (h). En síntesis, se observa que los esfuerzos de secado, inicialmente actúan provocando la contracción inicial en la superficie de la madera (figura 5a) y compresión hacia el centro de la madera, lo cual se revierte hacia el final del secado (Figura 5b), restando tensiones residuales de compresión en la superficie y tracción en el centro de la madera (Figura 5c).

En particular, en la figura 16a, representativa de la fase inicial de secado, se observa que la sección $\mathrm{N}^{\circ} 7$ tiene una deformación de $0.05 \%$ a diferencia de la sección $\mathrm{N}^{\mathrm{o}} 1$ que presenta una deformación de $0.4 \%$ (ambas secciones corresponden a la superficie y están sometidas a convección). Dicha diferencia disminuye conforme avanza el proceso de secado estableciéndose en un $0.8 \%$ a las 120 (h). La falta de simetría del comportamiento se explica debido que la convección en la sección $\mathrm{N}^{\circ} 7$ se ve afectada por su mayor cercanía a la zona de humectación (AS más húmedo). Además, se puede observar que tanto la sección $\mathrm{N}^{\circ} 1$ como la $\mathrm{N}^{\circ}$, en las etapas iniciales de secado, se contraen a mayor velocidad en la superficie, dando origen a contracciones $(\varepsilon<0)$ hasta las 96 (h) y a extensiones en $(\varepsilon>0)$ etapas finales de secado (120 (h)), lo cual se revierte hacia el final de secado. Este comportamiento se produce de forma inversa en el interior de la madera. En conclusión, los resultados experimentales muestran un comportamiento esperado en cuanto a la dinámica de tensión/compresión que experimentan las diversas regiones de la madera durante el proceso de secado. Siendo así, se espera que las deformaciones y esfuerzos determinados, a partir de estos ensayos, sean representativos del fenómeno estudiado y sirvan efectivamente al propósito de validar simulaciones.

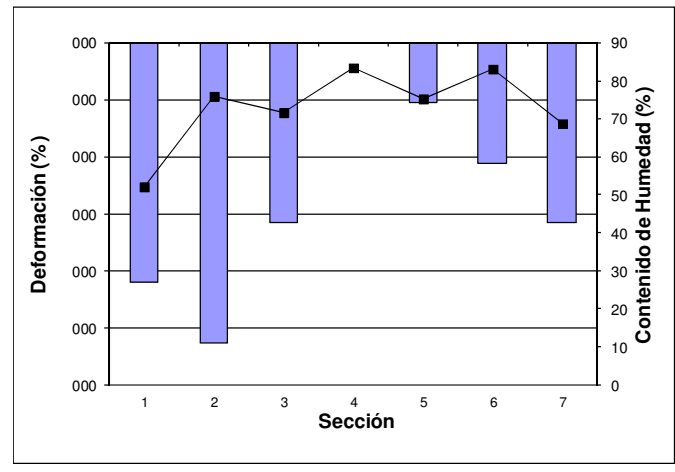

a) $24(\mathrm{~h})$

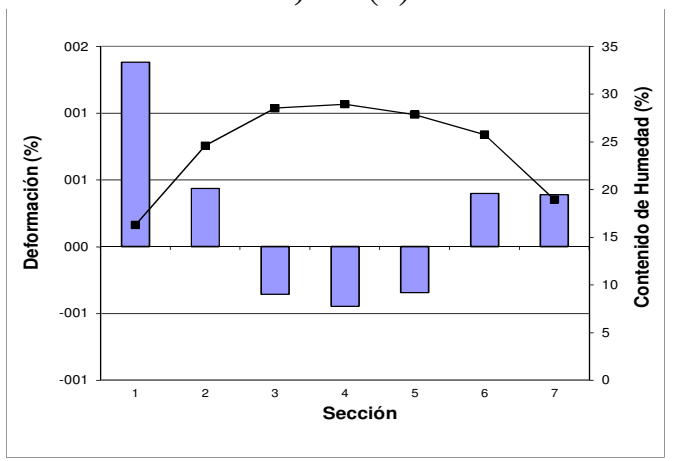

96 (h)

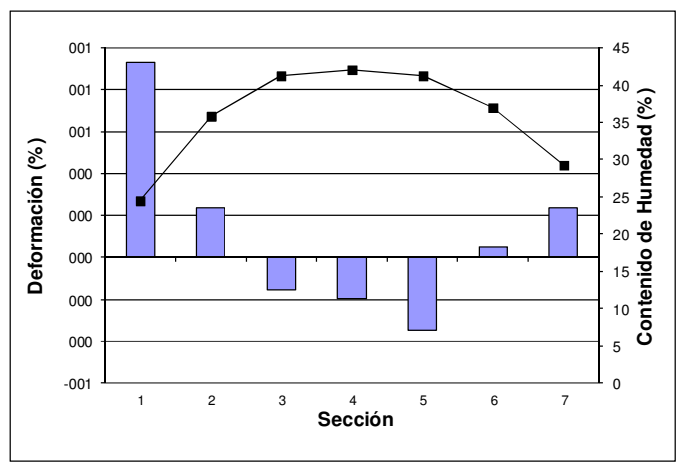

b) $72(\mathrm{~h})$

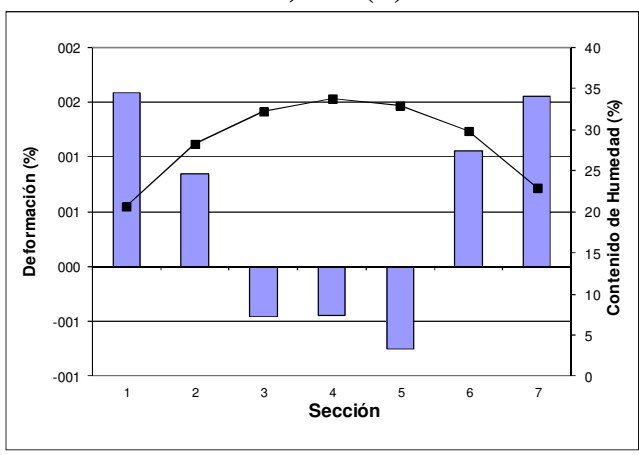

d) $120(\mathrm{~h})$

Figura 5. Deformación vs contenido de humedad. 
El comportamiento de los esfuerzos a través del tiempo es dado en la figura 6: Figura 6a y $6 \mathrm{~b}$ muestra los esfuerzos normales en las secciones $\mathrm{N}^{\circ} 1$ a $\mathrm{N}^{\circ} 4$ y No 4 a No 7 , respectivamente. En esta se destaca el comportamiento de la superficie de la pieza de madera (sección $\mathrm{N}^{\mathrm{o}} 1$ y 7) que comienza su estado de esfuerzos en tensión y al final del secado presenta un estado de compresión (sección $\mathrm{N}^{\mathrm{o}} 4$ ). En particular, en la superficie se aprecia una contracción de la madera al inicio del secado $(24$ h), lo que induce esfuerzos de tensión debido a la restricción impuestas por las capas adyacentes a la superficie, no evidenciándose deformaciones en el centro de la madera. A las 48 (h) se observa una expansión pequeña originando esfuerzos de compresión en las capas adyacentes al centro, y desde las 72 hasta las 120 (h) de secado se invierten los esfuerzos hacia las etapas finales de secado.

En síntesis, la figura 6 muestra el comportamiento cualitativo, con valores de esfuerzos en el rango de 0 hasta $3 \mathrm{MPa}$, concordantes con la bibliografía (Pang 2000).

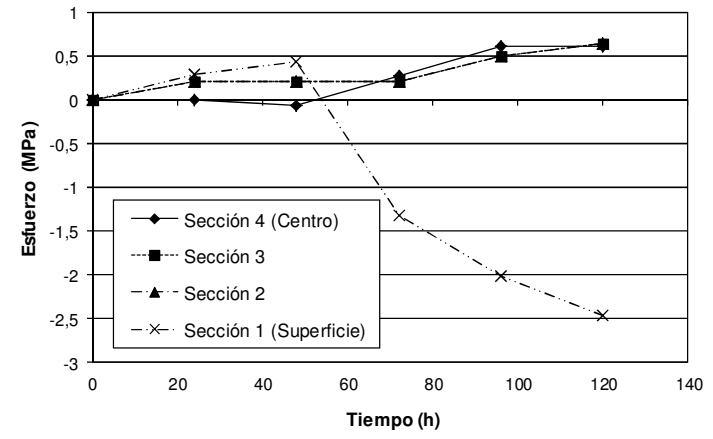

a) secciones $1-4$

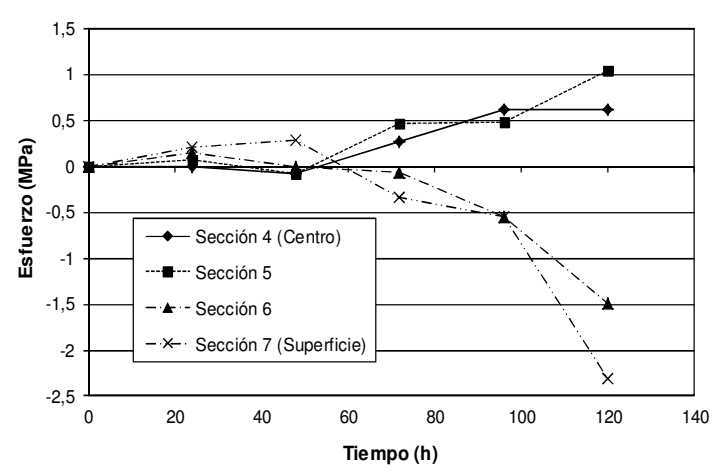

b) secciones 4-7

Figura 6. Esfuerzos normales transitorios de secado.

\subsection{Simulación}

Resultados de las simulaciones de esfuerzos de secado son mostrados en las figuras 7 . En particular, la figura 7a muestran los esfuerzos y contenidos de humedad media transitorios. En ellos se puede observar que su comportamiento cualitativo es el esperado: desarrollo de tensiones contrapuestas entre la superficie y el centro de la pieza de madera, conforme restricción de extensión y contracción de la madera, según discusión de resultados experimentales dados en la figura 5 y 6 . En términos de magnitud, los esfuerzos simulados se encuentran en los rangos característicos determinados experimentalmente, -2.5 a $2 \mathrm{MPa}$. Las figuras 7b y 7c corresponden a simulaciones transitorias que describen los esfuerzos de secado en el dominio de cálculo. En la primera etapa de secado (figura 7b), los esfuerzos simulados presentan un estado de tensión en las superficies de la madera expuesta a convección, los cuales están bien correlacionados con lo observado experimentalmente en las secciones $\mathrm{N}^{\circ} 1, \mathrm{~N}^{\circ} 2, \mathrm{~N}^{\circ} 6$ y $\mathrm{N}^{\circ} 7$, a su vez, en el centro de la madera, los esfuerzos comienzan a actuar en compresión, lo cual se correlaciona bien con las secciones $\mathrm{N}^{\circ} 3, \mathrm{~N}^{\circ} 4, \mathrm{~N}^{\circ}$. El avance del frente de secado induce el cambio de sentido en el estado de esfuerzos, por esta razón los esfuerzos en la segunda etapa o final de secado (figura 7c) presentan un estado de compresión cercanos a la superficie y tensión hacia el centro de la madera. Si bien es cierto el comportamiento cualitativo de las 
simulaciones se correlaciona bien con lo observado experimentalmente y que los órdenes de grandezas de las deformaciones residuales son semejantes, no es menos cierto que existen diferencias sustantivas en su desarrollo transitorio. Lo anterior, puede ser explicado por las limitaciones experimentales para obtener distribuciones transitorias de humedad y de esfuerzos durante el secado, así como, con una correcta simulación del transporte de humedad. Esta última, variable determinante en el modelo de esfuerzos de secado.

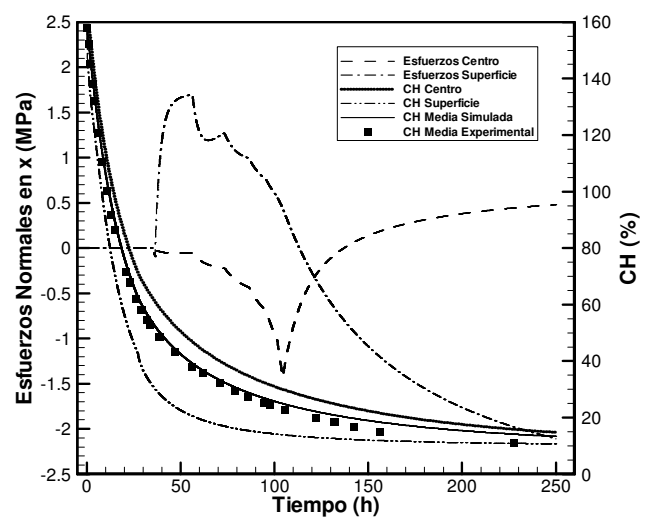

a) $\mathrm{CH}$ y $\sigma$

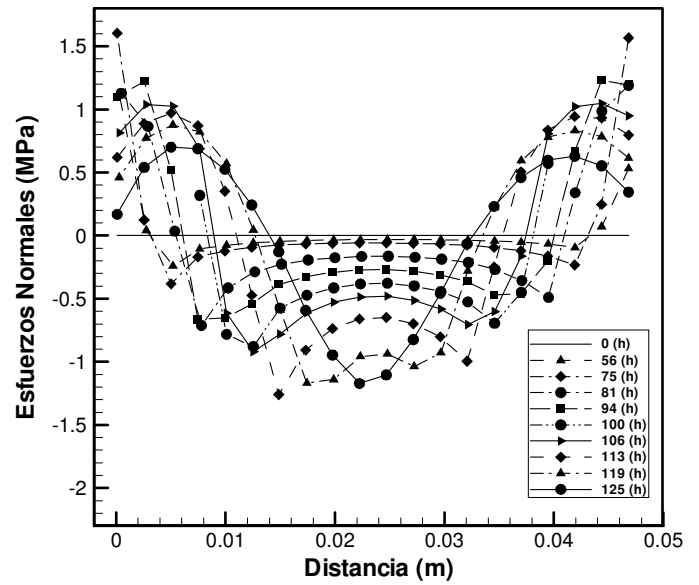

b) $\sigma, 1^{\circ}$ Etapa

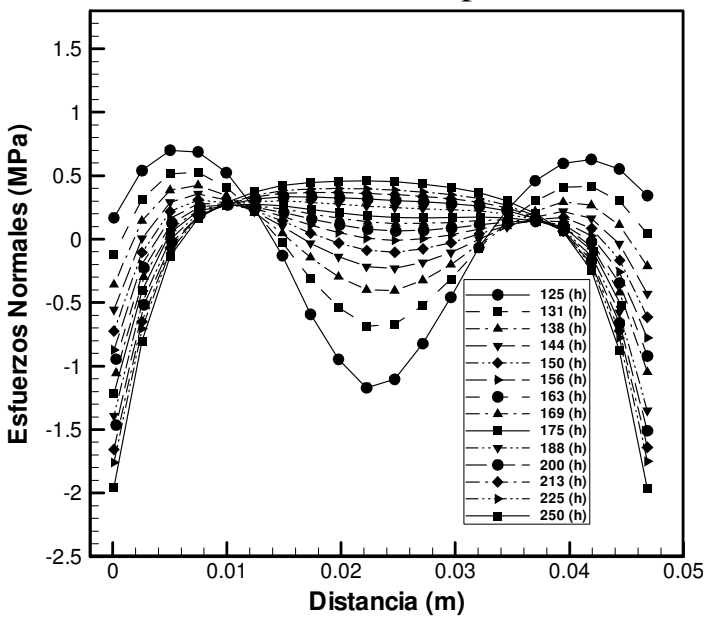

c) $\sigma, 2^{\circ}$ Etapa

Figura 6. Esfuerzos normales transitorios de secado. 


\section{CONCLUSIONES}

Se ha mostrado una metodología efectiva que permite colectar datos experimentales y simular esfuerzos durante el proceso de secado tradicional de madera de Pinus radiata.

El modelo experimental de transporte $\mathrm{CH}$ muestra datos consistentes, en cuanto al comportamiento cualitativo y cuantitativo del transporte de $\mathrm{CH}$ en madera de Pinus radiata. De la misma forma, el modelo experimental para el fenómeno tensión/deformación permite obtener datos consistentes de tensiones durante el proceso de secado. Esto es, esfuerzos de tensión en la superficie y compresión al interior de la madera al inicio del secado y un comportamiento reciproco hacia las etapas finales del secado.

Se ha modelado y simulado en forma efectiva (desviaciones $<3 \%$ ) procesos de transporte transitorio del secado tradicional de madera sólida, en base al concepto de Difusión Efectiva. De la misma forma, se ha modelado y simulado esfuerzos transitorios de secado, inducidos por gradientes de humedad, correlacionados con datos experimentales originales. Para este caso, los resultados son cualitativamente buenos, con valores dentro de rangos característicos determinados experimentalmente: -2.5 a 2 (MPa). Sin embargo, la evolución transitoria de esfuerzos presenta valores simulados con importantes desviaciones respecto de los experimentales.

\section{SIMBOLOGÍA}

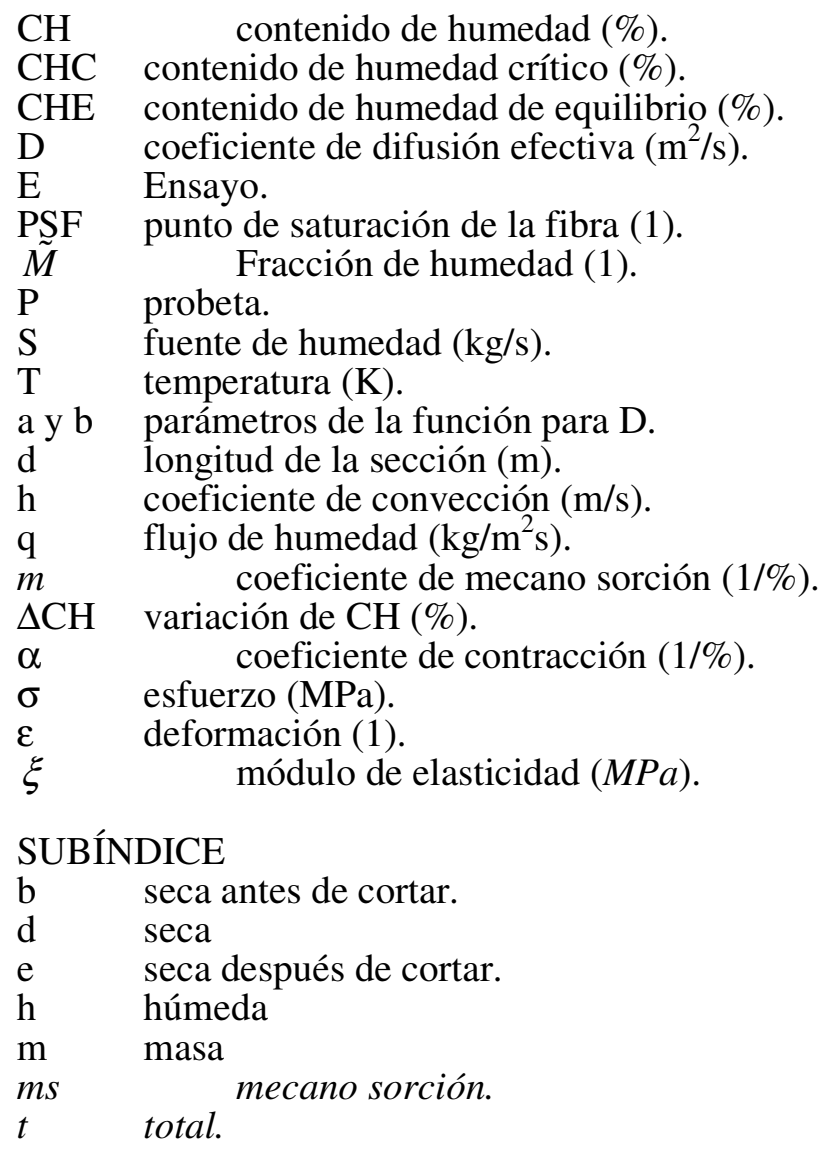




$\begin{array}{ll}s & \text { superficie } \\ \mathrm{v} & \text { verde } \\ x & \text { deformación libre } \\ \alpha & \text { contracción. } \\ \xi & \text { elástico. }\end{array}$

\section{REFERENCIAS}

[1] ...BALIGA, B.R.; PATANKAR, S.V. 1980. A new finite element formulation for convection diffusion problems. Numerical Heat Transfer 3:393-409.

[2] ...BURH, H., STAMM, A. 1947. Diffusion in wood. J Phys Colloid Chem. 51(1):240-61.

[3] CHÁVEZ, C. 2009. Simulación bidimensional de los esfuerzos de secado en la madera a través de CVFEM. Tesis de Magister en Ciencia y Tecnología de la Madera, Concepción, Facultad de Ingeniería, Universidad del Bio Bio, Chile.

[4] ...CHEN, X.D. 2007 Moisture diffusivity in food and biological materials. Drying Technology, 25, 1203-1213.

[5] ...FERGUSON, W. J. 1998. The control volume finite element numerical solution technique applied to Creep in softwoods. Int. J. Solid Structures 35(13):1325-1338.

[6] ...SAlinAS, C.H., GATICA, Y.A.; ANANIAS, R.A. 2012. "Modeling conventional two-dimensional drying of radiata pine based on trransversal diffusion coefficiennt". Accepted for publication in Lat Am Appl Res.

[7] ...GATICA, Y. A., SAlinAS, C. H.; ANANIAS, R. A. 2011. Modelling Conventional One-Dimensional Drying of Radiata Pine Based on the Effective Diffusion Coefficient. Latin American Applied Research. Vol 41, № 2, 183-189.

[8] HUKKA, A. 1999. The effective diffusion coefficient and mass transfer coefficient of nordic softwoods as calculated from direct drying experiments. Holzforschung, 53, 534540 .

[9] KANG W., LEE ., JUNG H. 2004. Simple analytical methods to predict one-an twodimensional drying stresses and deformations in lumber. Wood Sci. Technol. 38 417-428.

[10] KEEY R.; LANGRISH T.; WALKER J. 2000. Kiln-drying of lumber. Springerverlag, Berlin, pp. 65-115, 175-181.

[11] MCMILLEN J. 1963. Stresses in wood during drying. Res. Rap. 1652 USDA Forest Service. Forest Products Lab. Madison, WI.

[12] MOREN, T; SEHLSTEDT-PERSSON, M. 1992. Creep deformation of the surface layer of timber board during air circulation drying. $3^{\text {RD }}$ IUFRO Drying conference, Vienna, pp 96-102.

[13] ORMARSSON, S.; DAHLBLOM, O.; JOHANSSON, M. 2009. Finite element study of growth stress formation in wood and related distortion of sawn timber. Wood Science and Technology, vol. 43, pp 387-403.

[14] ORMARSSON, S., DAHLBLOM O., PETERSSON H., 1998. A numerical study of the shape stability of sawn timber subjected to moisture variation. Part 1: Theory. Wood 
Science and Technology, vol 32, pp 325-334.

[15] PANG S. 2000. Modelling of stress development during drying and relief during steaming in Pinus radiate lumber. Drying Technology 18(8):1677-1696.

[16] RÉMOND R.; PERRE, 2008. Drying strategies capable of reducing the streess level of a stack of boards as defined by a comprehensive dual scale model. Maderas, Ciencia y Tecnología 10(1): 3-18.

[17] ROZAS, C.; TOMASELLI, I.; ZANOELO, E. 2009. Internal mass transfer coefficient during drying of softwood (Pinus elliotti) boards. Wood Science and Technology 43:361-373.

[18] SALINAS, C. R. ANANIAS M. ALVEAR. 2004. Simulación del secado convencional de la madera. Maderas Ciencia y Tecnología 6(1): 3-18.

[19] SIMPSON, W.T; LIU J.Y. 1997 An optimization technique to determine red oak surface and internal moisture transfer coefficients during drying. Wood and Fiber Science, 29 (4), 312-318.

[20] STEVENSSON, S.; MARTENSSON, A. 2002. Simulation of drying stresses in wood Part II Convective air drying of sawn timber. Holz Roh Werkst. 60:72-80.

AGRADECIMIENTOS: Fondecyt, proyecto $\mathbf{N}^{\circ} 1110500$. 